Bull. Austral. Math. Soc.

Vol. 53 (1996) [499-515]

\title{
BOUNDS ON THE LIMITING DISTRIBUTION OF A BRANCHING PROCESS WITH VARYING ENVIRONMENT
}

\section{OWEN DAFYDD JONES}

Upper and lower bounds are obtained for the left tail of the normed limit $W_{0}$ of a supercritical branching process with varying environment, that is, for $\mathrm{P}\left(W_{0}<x\right)$ for small $x$. Two types of process are dealt with--Böttcher type and Schröder type-which between them cover "most" processes with zero extinction probability.

\section{INTRODUCTION}

A single-type branching process in a varying environment generalises the classical branching process (or Galton-Watson process), in that the number of offspring born to any individual depends on that individual's generation. Let the offspring distribution of an individual at time $n$ be given by the law of

$$
X_{n} \quad n=0,1,2, \ldots
$$

and denote by

$$
Z_{m, n} \quad n=m, m+1, m+2, \ldots
$$

the total number of descendants at time $n$ of a single parent at time $m$. Let $\mu_{n}=\mathrm{E} X_{n}$, then $\mu_{m, n}:=\prod_{k=m}^{n-1} \mu_{k}=\mathrm{E} Z_{m, n}$. We shall assume the $\mu_{n}$ are finite throughout. Put

$$
W_{m, n}=Z_{m, n} / \mu_{m, n} .
$$

The following results are well known:

TheOREM 1. (Almost surely convergence. Fearn [11], Jagers [15].) $\left\{W_{m, n}\right\}_{n=m}^{\infty}$ is a non-negative martingale with respect to the filtration $\left\{\mathcal{F}_{m}^{n}\right\}_{n=m}^{\infty}$, where $\mathcal{F}_{m}^{n}$ is the $\sigma$-algebra generated by $\left\{Z_{m, m}, Z_{m, m+1}, \ldots, Z_{m, n}\right\}$. Thus the $W_{m, n}$ converge almost surely to a random variable $W_{m}$ with $\mathrm{E} W_{m} \leqslant 1$.

TheOREM 2. ( $\mathcal{L}^{2}$ convergence. Fearn [11], Jagers [15].) Suppose that $v_{n}:=$ $\operatorname{Var}\left(X_{n} / \mu_{n}\right)$ exists and is finite for all $n$. Then if

$$
\sum_{k=m}^{\infty} \frac{v_{k}}{\mu_{m, k}}<\infty
$$

Received 2nd March, 1995

The author would like to thank John Biggins for his valuable comments on an earlier version of this paper. Research partially funded by U.K. EPSRC grant no. GR/J 76187.

Copyright Clearance Centre, Inc. Serial-fee code: 0004-9729/96 \$A2.00+0.00. 
then $W_{m, n} \stackrel{\mathcal{L}^{2}}{\longrightarrow} W_{m}$ and hence $\mathrm{E} W_{m}=1$.

Note that $\operatorname{Var} W_{m, n}=\sum_{k=m}^{n-1} v_{k} / \mu_{m, k}$, so (1) is equivalent to

$$
\lim _{n \rightarrow \infty} \operatorname{Var} W_{m, n}<\infty
$$

Moreover, it follows that $\operatorname{Var} W_{m}$ exists and equals $\sum_{k=m}^{\infty} v_{k} / \mu_{m, k}$.

More recently, D'Souza and Biggins [8] have provided a generalisation of one half of the classic result of Kesten and Stigum [16]. Say the process $Z_{m, n}$ (for fixed $m$ ) is uniformly supercritical if there exist constants $A>0$ and $c>1$ such that for all $n \geqslant m$

$$
\mu_{m, n} \geqslant A c^{n-m} .
$$

Also, say that the random variable $X$ dominates $Y$ if for all $x$

$$
\mathrm{P}(Y>x) \leqslant \mathrm{P}(X>x) .
$$

Given these definitions we have:

Theorem 3. (Single growth rate. D'Souza and Biggins [8].) If the process $Z_{m, n}$ is uniformly supercritical and $X_{n} / \mu_{n}$ is dominated by some $X$ for all $n \geqslant m$, where

$$
\mathrm{E} X \log ^{+} X<\infty,
$$

then $\mathrm{E} W_{m}=1$ and $\left\{W_{m}>0\right\}=\left\{Z_{m, n} \rightarrow \infty\right\}$ almost surely.

Note that the conditions of Theorem 2 are not sufficient to give $\left\{W_{m}>0\right\}=$ $\left\{Z_{m, n} \rightarrow \infty\right\}$ almost surely, as is shown by the example of MacPhee and Schuh [17]. In particular, the conditions of Theorem 2 do not imply those of Theorem 3. (A simple condition, sufficient to imply the conditions of both Theorems 2 and 3 , is that $1<\underline{\mu}:=\liminf _{n} \mu_{n} ; \bar{\mu}:=\sup _{n} \mu_{n}<\infty$ and $\bar{v}:=\sup _{n} v_{n}<\infty$. A suitable dominating random variable in this case is given by $X$ with density $2 k^{2} x^{-3}$ on $[k, \infty)$, where $k^{2}>\bar{v}+\bar{\mu}^{2}$.) Conditions for $\mathrm{E} W_{m}=1$, strictly weaker than those of Theorem 3, are given by Goettge [12]. However, these are again insufficient to give a single rate of growth, as is shown in D'Souza and Biggins [8, Section 4]. Moreover, when $Z_{m, n}$ is uniformly supercritical, Goettge's conditions are in practice the same as those of D'Souza and Biggins. See Goettge's Theorems 5 and 7 and Example 9.

For the classical branching process we have $X_{n}=X$ for all $n$, and so $W_{m}=$ $W$ for all $m$. Analysis of $W$ has distinguished two cases. We call the branching process Schröder if $\mathrm{P}(X=0)=0$ and $\mathrm{P}(X=1)>0$, and Böttcher if $\mathrm{P}(X=0)=$ 
$\mathrm{P}(X=1)=0$. In each case there is a single parameter which describes the asymptotic distribution of $W$ at the origin. Put $\mu=\mathrm{E} X$, then in the Schröder case we have, putting $p_{1}=\mathrm{P}(X=1)$

$$
\alpha=\frac{-\log p_{1}}{\log \mu}
$$

and for the Böttcher case, putting $a=\min \{k: \mathrm{P}(X=k)>0\}$

$$
\gamma=\frac{\log a}{\log \mu} .
$$

In the Schröder case Dubuc $[9,10]$ obtained the following algebraic bounds on the law of $W$ : for all $0 \leqslant x \leqslant x_{0}$ (some $x_{0}>0$ )

$$
c_{1} x^{\alpha} \leqslant \mathrm{P}(W<x) \leqslant c_{2} x^{\alpha} .
$$

(Dubuc in fact gives bounds on the density of $W$. Integrating these gives the bounds on the law of $W$ referred to.) In the Böttcher case Dubuc [10] and Bingham [7] obtained the following exponential bounds: for all $x \geqslant 0$

$$
c_{3} \exp \left\{-c_{4} x^{-\gamma /(1-\gamma)}\right\} \leqslant \mathrm{P}(W<x) \leqslant c_{5} \exp \left\{-c_{6} x^{-\gamma /(1-\gamma)}\right\} .
$$

Here $c_{1}, \ldots, c_{6}$ are positive constants. Analogous results for the random environment case have been proved by Hambly [13]. In what follows we generalise these results to branching processes with varying environments. That is, we construct upper and lower bounds for the left tail of $W_{m}$. The same two cases appear. Accordingly, say $Z_{m, n}$ is of Schröder type if $X_{n}$ is Schröder for all $n \geqslant m$ and of Böttcher type if $X_{n}$ is Böttcher for all $n \geqslant m$. Our results appear as Propositions 6 and 9 for the Böttcher case and as Propositions 12 and 14 for the Schröder case.

We shall take as our basic assumptions:

C1 $\bar{\mu}:=\sup \mu_{n}<\infty$.

C2 $Z_{0, n}$ is uniformly supercritical.

C3 All the $X_{n} / \mu_{n}$ are dominated by some $X$ with $\mathrm{E} X \log ^{+} X<\infty$.

Conditions $\mathrm{C} 2$ and $\mathrm{C} 3$ are the conditions of Theorem 3. Trivially, C2 is always satisfied in the Böttcher case. In both the Schröder and Böttcher cases, the upper bounds we find have in common with Theorem 3 a fundamental lemma, which uses the uniform moment condition C3: see Lemma 4 below and Proposition 2 of D'Souza and Biggins [8]. Our lower bounds do not use Theorem 3 directly, though they do require $\mathrm{E} W_{m}=$ 1. However, as we shall be assuming uniform supercriticality, this condition is (given current technology) essentially the same as C3. 
Condition $\mathrm{C} 1$ can be thought of as complementing $\mathrm{C} 2$.

The initial motivation for this work came from the study of diffusions on fractals. It turns out that in many cases a diffusion can be constructed on a fractal using a "nested" sequence of random walks, which has associated with it a branching process. Bounds on the law of the normed limit of this branching process translate directly into sample path results for the corresponding diffusion, and so are of some interest. For a review of the literature in this area see Barlow [1, 2]. In addition, these branching processes exhibit near-constancy phenomena in the limit. A number of results in this area have been given by Biggins and Bingham $[4,5]$. These involve (amongst other things) bounds on both the left and right tails, though under different assumptions, providing generalisations of the above results for the classical branching process. Hambly [14] is also of interest in this context, as it gives an explicit calculation of the density of $W$ in a special case.

The methods we use owe much to those of Hambly [13] and are similar to the arguments used by Barlow and Bass [3, Lemma 4.4].

\section{Preliminaries}

For $t \in[0,1]$, let $f_{n}(t)=\mathrm{E} t^{X_{n}}$ be the probability generating function of $X_{n}$ and $f_{m, n}(t)=\mathrm{E} t^{Z_{m, n}}$ the probability generating function of $Z_{m, n}$, then conditioning on $Z_{m, m+1}$

$$
\begin{aligned}
f_{m, n}(t) & =f_{m}\left(f_{m+1, n}(t)\right) \\
& =f_{m}\left(f_{m+1}\left(\cdots\left(f_{n-1}(t)\right) \cdots\right)\right) .
\end{aligned}
$$

For $u \in \mathbf{R}_{+}$, write $\varphi_{m, n}(u)=\mathrm{E} e^{-u W_{m, n}}$ for the Laplace transform of $W_{m, n}$ and $\varphi_{m}(u)=\mathbf{E} e^{-u W_{m}}$ for the Laplace transform of $W_{m}$, then as $W_{m, n} \stackrel{\text { a.s. }}{\longrightarrow} W_{m}, \varphi_{m, n}(u) \rightarrow$ $\varphi_{m}(u)$. Moreover from (2), putting $t=e^{-u / \mu_{m, p}}$ in $f_{m, p}(t)$, we get

$$
\varphi_{m, p}(u)=f_{m, n}\left(\varphi_{n, p}\left(u / \mu_{m, n}\right)\right)
$$

and so letting $p \rightarrow \infty$

$$
\varphi_{m}(u)=f_{m, n}\left(\varphi_{n}\left(u / \mu_{m, n}\right)\right)
$$

Let $p_{i}^{n}=\mathrm{P}\left(X_{n}=i\right), p_{i}^{m, n}=\mathrm{P}\left(Z_{m, n}=i\right), a_{n}=\inf \left\{i: p_{i}^{n}>0\right\}$ and $a_{m, n}=$ $\inf \left\{i: p_{i}^{m, n}>0\right\}=\prod_{k=m}^{n-1} a_{k}$. Then

$$
\begin{aligned}
f_{m, n}(t) & =\sum_{i=a_{m, n}}^{\infty} p_{i}^{m, n} t^{i} \\
& =t^{a_{m, n}} \sum_{i=a_{m, n}}^{\infty} p_{i}^{m, n} t^{i-a_{m, n}}
\end{aligned}
$$


whence, as $\sum_{i=a_{m, n}}^{\infty} p_{i}^{m, n} t^{i-a_{m, n}} \leqslant 1$ (as it is a probability generating function)

$$
p_{a_{m, n}}^{m, n} t^{a_{m, n}} \leqslant f_{m, n}(t) \leqslant t^{a_{m, n}}
$$

Substituting (3) into (4) gives for all $n \geqslant m \geqslant 0$

$$
p_{a_{m, n}}^{m, n} \varphi_{n}\left(u / \mu_{m, n}\right)^{a_{m, n}} \leqslant \varphi_{m}(u) \leqslant \varphi_{n}\left(u / \mu_{m, n}\right)^{a_{m, n}} .
$$

This inequality is the foundation upon which our results rest.

\section{BötTCher Case}

We shall assume throughout this section that $Z_{0, n}$ is Böttcher. The varying environment analogue to $\gamma$ is given by two parameters. For any $m \geqslant 0$ define

$$
\gamma^{-}:=\liminf _{n \rightarrow \infty} \frac{\log a_{m, n}}{\log \mu_{m, n}} \quad \text { and } \quad \gamma^{+}:=\limsup _{n \rightarrow \infty} \frac{\log a_{m, n}}{\log \mu_{m, n}} .
$$

It is easily checked that $\gamma^{-}$and $\gamma^{+}$do not depend on $m$, and that

$$
\liminf _{n \rightarrow \infty} \frac{\log a_{n}}{\log \mu_{n}} \leqslant \gamma^{-} \leqslant \gamma^{+} \leqslant \limsup _{n \rightarrow \infty} \frac{\log a_{n}}{\log \mu_{n}} .
$$

Also note that it is possible to have $\gamma^{-}<\gamma^{+}$. We shall write

$$
\gamma_{\varepsilon}^{+} \text {for } \gamma^{+}+\varepsilon \text { and } \gamma_{\varepsilon}^{-} \text {for } \gamma^{-}-\varepsilon
$$

Observe that $\mathrm{Cl}$ implies $\gamma^{-}>0$. However, to guarantee $\gamma^{+}<1$ will require further conditions.

\subsection{UPPER BOUND.}

An upper bound on the law of $W_{0}$ is obtained from an upper bound on $\varphi_{0}$. To bound $\varphi_{0}$ we firstly get a uniform bound on all the $\varphi_{n}$ near 0 , and then apply (5) to these, to extend the bound on $\varphi_{0}$ out to infinity. (Hambly [13, Section 3] gives some discussion of the reasoning behind this approach.)

LEmma 4. (Uniform upper bound for all $\varphi_{n}$.) Suppose $C 2$ and $C 3$ hold, then for any $u_{0}>0$ there exists a $\beta<1$ such that for all $n$

$$
\varphi_{n}(u) \leqslant \beta \quad \text { for all } u \geqslant u_{0}
$$

Proof: Condition C2 gives us constants $A>0$ and $c>1$ such that $\mu_{0, n} \geqslant A c^{n}$ for all $n \geqslant 0$. From Proposition 2 of D'Souza and Biggins [8] there exists a $\theta_{0}>0$ such that for all $0 \leqslant \theta \leqslant A \theta_{0}$

$$
\varphi_{n}(\theta) \leqslant e^{-\theta}+\theta r(\theta)+\frac{\theta}{\log c} \int_{0}^{\theta / A} \frac{r(\omega)}{\omega} d \omega
$$


where, if $X$ is the dominating random variable from Condition C3

$$
r(\theta)=\frac{1}{\theta} E\left[e^{-\theta X}-1+\theta X\right]
$$

D'Souza and Biggins also note that both $r(\theta)$ and $\int_{0}^{\theta / A}(r(\omega) / \omega) d \omega$ tend to 0 as $\theta \rightarrow 0$. Thus for any $\varepsilon>0$ we can find a $\delta$ such that for all $0 \leqslant \theta \leqslant \delta$

$$
r(\theta)+\frac{1}{\log c} \int_{0}^{\theta / A} \frac{r(\omega)}{\omega} d \omega \leqslant \varepsilon
$$

whence $\varphi_{n}(\theta) \leqslant e^{-\theta}+\theta \varepsilon$. Take $\varepsilon<1$, then, noting that $\varphi_{n}$ is decreasing, we have for all $\theta \geqslant 0$

$$
\varphi_{n}(\theta) \leqslant s(\theta):= \begin{cases}e^{-\theta}+\theta \varepsilon & \text { for } 0 \leqslant \theta \leqslant-\log \varepsilon \\ \varepsilon-\varepsilon \log \varepsilon & \text { for }-\log \varepsilon \leqslant \theta<\infty .\end{cases}
$$

Observe that $\varepsilon-\varepsilon \log \varepsilon<1$ for $\varepsilon<1$, then the result follows on putting $\beta=s\left(u_{0}\right)$.

Note that Lemma 4 does not require $Z_{0, n}$ to be Böttcher, and will be used again in the next section.

Proposition 5. (Exponential upper bound for $\varphi_{0}$.) Suppose that C1-C3 hold, then there exists a $c_{1}>0$ such that for all $0<\varepsilon<\gamma^{-}$we can find a $u_{1}$ such that for all $u>u_{1}$

$$
\varphi_{0}(u) \leqslant \exp \left\{-c_{1} u^{\gamma_{e}^{-}}\right\}
$$

Proof: Let $u_{0}=1 / \bar{\mu}$. Define intervals

$$
\begin{aligned}
& I_{0}=\left[u_{0}, 1\right] \text { and } \\
& I_{n}=\left(\mu_{0, n-1}, \mu_{0, n}\right] \text { for } n>0 .
\end{aligned}
$$

Then for $u \in I_{n}$ we have $u / \mu_{0, n} \in I_{0}$, whence from (5) and (6)

$$
\begin{aligned}
\varphi_{0}(u) & \leqslant \varphi_{n}\left(u / \mu_{0, n}\right)^{a_{0, n}} \\
& \leqslant \beta^{a_{0, n}} \\
& =\exp \left\{-\log \left(\beta^{-1}\right) \mu_{0, n}^{\log a_{0, n} / \log \mu_{0, n}}\right\} \\
& \leqslant \exp \left\{-\log \left(\beta^{-1}\right) u^{\log a_{0, n} / \log \mu_{0, n}}\right\}
\end{aligned}
$$

since $\mu_{0, n} \geqslant u$. Now, given $\varepsilon>0$, let $N$ be such that for all $n \geqslant N$

$$
\frac{\log a_{0, n}}{\log \mu_{0, n}} \geqslant \gamma_{\varepsilon}^{-}
$$


Then for $u>\mu_{0, N-1}:=u_{1}$ we have $n \geqslant N$ and so

$$
\varphi_{0}(u) \leqslant \exp \left\{-\log \left(\beta^{-1}\right) u^{\gamma_{\bar{\varepsilon}}^{-}}\right\} .
$$

Putting $c_{1}=\log \left(\beta^{-1}\right)>0$ gives the result.

Note that if $\gamma^{-}=\inf _{n} \log a_{0, n} / \log \mu_{0, n}$, then the result holds with $\varepsilon=0$ and $u_{1}=u_{0}$.

Proposition 6. (Exponential upper bound on the law of $W_{0}$.) Suppose that C1-C3 hold, then there exists a $c_{2}>0$ such that for all $0<\varepsilon<\gamma^{-}$we can find an $x_{0}$ such that for all $0 \leqslant x<x_{0}$

$$
\mathrm{P}\left(W_{0}<x\right) \leqslant \exp \left\{-c_{2} x^{-\gamma_{e}^{-}} /\left(1-\gamma_{e}^{-}\right)\right\} .
$$

Proof: Markov's inequality gives

$$
\begin{aligned}
\mathrm{P}\left(W_{0}<x\right) & =\mathrm{P}\left(e^{-u W_{0}}>e^{-u x}\right) \\
& \leqslant e^{u x} \varphi_{0}(u) \\
& \leqslant \exp \left\{u x-c_{1} u^{\gamma_{e}^{-}}\right\} \text {from (7) }
\end{aligned}
$$

(Inequalities of the form $\mathrm{P}\left(W_{0}<x\right) \leqslant e^{u x} \varphi_{0}(u)$ are commonly found in the large deviations literature. In particular, an inequality of this sort (proved in the same way) is the starting point of Chernoff's Theorem. See for example Billingsley [6, Section 9].)

Now $u x-c_{1} u^{\gamma_{e}^{-}}$has a minimum of

$$
-c_{2} x^{-\gamma_{e}^{-} /\left(1-\gamma_{e}^{-}\right)}
$$

occurring at

$$
u=\left(\frac{x}{c_{1} \gamma_{e}^{-}}\right)^{1 /\left(\gamma_{e}^{-}-1\right)}
$$

where

$$
c_{2}=c_{1}^{1 /\left(1-\gamma_{\varepsilon}^{-}\right)}\left(\left[\gamma_{\varepsilon}^{-}\right]^{\gamma_{\varepsilon}^{-} /\left(1-\gamma_{\varepsilon}^{-}\right)}-\left[\gamma_{e}^{-}\right]^{1 /\left(1-\gamma_{e}^{-}\right)}\right)>0 .
$$

This $u$ is greater than $u_{1}$ so long as

$$
x<x_{0}:=u_{1}^{\gamma_{e}^{-}-1} c_{1} \gamma_{\epsilon}^{-} .
$$

Minimising $c_{2}$ over $0<\varepsilon<\gamma^{-}$now gives the result.

Again note that if $\gamma^{-}=\inf _{n} \log a_{0, n} / \log \mu_{0, n}$, then the result holds with $\varepsilon=0$ and $x_{0}=u_{0}^{\gamma^{-}-1} c_{1} \gamma^{-}$. 


\subsection{LOWER BOUND.}

For a lower bound on the law of $W_{0}$ we again proceed via a bound on $\varphi_{0}$. A lower bound on $\varphi_{0}$ is obtained from a rough but uniform lower bound on the $\varphi_{n}$, which is then refined by suitable application of (5). This clearly will require some additional estimates on $p_{a_{0, n}}^{0, n}$, for which we shall need some additional assumptions. Firstly, to guarantee $\gamma^{+}<1$ we need:

$$
\text { C4 } \bar{p}:=\sup _{n} p_{a_{n}}^{n}<1 .
$$

Secondly, for fixed $m$, say $Z_{m, n}$ has uniform minimum family sizes if there exist some $B, q>0$ such that

$$
p_{a_{m, n}}^{m, n} \geqslant B q^{a_{m, n}} \quad \text { for all } n \geqslant m
$$

We shall assume

C5 $Z_{0, n}$ has uniform minimum family sizes.

Note that C5 certainly holds if $\underline{p}:=\inf _{n} p_{a_{n}}^{n}>0$, since in the Böttcher case

$$
\begin{aligned}
p_{a_{0, n}}^{0, n} & =\prod_{k=0}^{n-1}\left(p_{a_{k}}^{k}\right)^{a_{0, k}} \\
& \geqslant \underline{p}^{\sum_{k=0}^{n-1} a_{0, k}} \\
& \geqslant \underline{p}^{a_{0, n}}
\end{aligned}
$$

upon noting that $\sum_{k=0}^{n-1} a_{0, k} \leqslant a_{0, n}$ (by induction, since $a_{n} \geqslant 2$ ). Also, if C5 holds then we can, by adjusting $q$ if necessary, assume $B=1$.

Lemma 7. (Uniform lower bound for all $\varphi_{n}$.) Suppose C2 and C3 hold, then

$$
\varphi_{n}(u) \geqslant e^{-u}
$$

for all $n$ and $u \geqslant 0$.

Proof: It follows from Theorem 3 that $\mathrm{E} W_{n}=1$ for all $n$. Thus from Jensen's inequality

$$
\varphi_{n}(u)=\mathrm{E} e^{-u W_{n}} \geqslant e^{-u E W_{n}}=e^{-u}
$$

as required.

Note that Lemma 7 does not require $Z_{0, n}$ to be Böttcher, and will be used again in the next section. 
Proposition 8. (Exponential lower bound for $\varphi_{0}$.) Suppose that C1-C5 hold, then there exists a $c_{1}>0$ such that for all $0<\varepsilon<1-\gamma^{+}$we can find a $u_{1}$ such that for all $u>u_{1}$

$$
\varphi_{0}(u) \geqslant \exp \left\{-c_{1} u^{\gamma_{e}^{+}}\right\}
$$

Proof: From (5) and C5 we have for all $n \geqslant 0$ (assuming $B=1$ )

$$
\begin{aligned}
\varphi_{0}(u) & \geqslant p_{a_{0, n}}^{0, n} \varphi_{n}\left(u / \mu_{0, n}\right)^{a_{0, n}} \\
& \geqslant\left(q \varphi_{n}\left(u / \mu_{0, n}\right)\right)^{a_{0, n}} \\
& \geqslant\left(q \exp \left\{-u / \mu_{0, n}\right\}\right)^{a_{0, n}} \quad \text { from (8) } \\
& =\exp \left\{-\left(u / \mu_{0, n}+\log (1 / q)\right) a_{0, n}\right\} .
\end{aligned}
$$

Define intervals

$$
\begin{aligned}
I_{0} & =[1, \bar{\mu}] \quad \text { and } \\
I_{n} & =\left(\bar{\mu} \mu_{0, n-1}, \bar{\mu} \mu_{0, n}\right] \quad \text { for } \quad n>0 .
\end{aligned}
$$

Then for $u \in I_{n}$ we have

$$
\begin{aligned}
\varphi_{0}(u) & \geqslant \exp \left\{-(\log (1 / q)+\bar{\mu}) a_{0, n}\right\} \\
& =\exp \left\{-(\log (1 / q)+\bar{\mu}) \mu_{0, n}^{\log a_{0, n} / \log \mu_{0, n}}\right\} \\
& \geqslant \exp \left\{-(\log (1 / q)+\bar{\mu}) u^{\log a_{0, n} / \log \mu_{0, n}}\right\} .
\end{aligned}
$$

Given $\varepsilon>0$ let $N$ be such that for all $n \geqslant N$

$$
\frac{\log a_{0, n}}{\log \mu_{0, n}} \leqslant \gamma_{e}^{+}
$$

then for all $u>\bar{\mu} \mu_{0, N-1}:=u_{1}$ we get $n \geqslant N$ and so

$$
\varphi_{0}(u) \geqslant \exp \left\{-c_{1} u^{\gamma_{e}^{+}}\right\}
$$

where $c_{1}=\log (1 / q)+\bar{\mu}$.

Note that if $\gamma^{+}=\sup _{n} \log a_{0, n} / \log \mu_{0, n}$, then the result holds with $\varepsilon=0$ and $u_{1}=1$.

Proposition 9. (Exponential lower bound on the law of $W_{0}$.) Suppose that C1-C5 all hold, then there exists a $c_{2}>0$ such that for all $0<\varepsilon<1-\gamma^{+}$we can find an $x_{0}$ such that for all $0 \leqslant x<x_{0}$

$$
\mathrm{P}\left(W_{0}<x\right) \geqslant \frac{1}{2} \exp \left\{-c_{2} x^{-\gamma_{e}^{+}} /\left(1-\gamma_{e}^{+}\right)\right\} .
$$


ProOF: Decomposing $\varphi_{0}$ gives

$$
\begin{aligned}
\varphi_{0}(u) & =\mathrm{E}\left(e^{-u W_{0}} I_{\left\{W_{0}<x\right\}}\right)+\mathrm{E}\left(e^{-u W_{0}} I_{\left\{W_{0} \geqslant x\right\}}\right) \\
& \leqslant \mathrm{P}\left(W_{0}<x\right)+e^{-u x}\left(1-\mathrm{P}\left(W_{0}<x\right)\right)
\end{aligned}
$$

whence from (9)

$$
\begin{aligned}
\mathrm{P}\left(W_{0}<x\right) & \geqslant \frac{\varphi_{0}(u)-e^{-u x}}{1-e^{-u x}} \\
& \geqslant \frac{\exp \left\{-c_{1} u^{\gamma_{e}^{+}}\right\}-\exp \{-u x\}}{1-\exp \{-u x\}} \\
& =\exp \left\{-c_{1} u^{\gamma_{c}^{+}}\right\} \frac{1-\exp \left\{c_{1} u^{\gamma_{c}^{+}}-u x\right\}}{1-\exp \{-u x\}} .
\end{aligned}
$$

Put $u=c_{0} x^{1 /\left(\gamma_{e}^{+}-1\right)}$ for some $c_{0}$, then $u>u_{1}$ if and only if $x<\left(u_{1} / c_{0}\right)^{\gamma_{e}^{+}-1}:=x_{0}$. Given this, we have

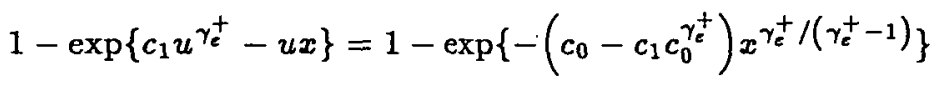

and

$$
1-\exp \{-u x\}=1-\exp \left\{-c_{0} x^{\gamma_{e}^{+}} /\left(\gamma_{e}^{+}-1\right)\right\}
$$

Choose $c_{0}$ so that $c_{0}-c_{1} c_{0}^{\gamma_{e}^{+}}=c_{0} / 2$; that is

$$
c_{0}=\left(2 c_{1}\right)^{1 /\left(1-\gamma_{\varepsilon}^{+}\right)} .
$$

Then

$$
\begin{aligned}
\frac{1-\exp \left\{c_{1} u^{\gamma_{e}^{+}}-u x\right\}}{1-\exp \{-u x\}} & \left.=\frac{1-\exp \left\{-\frac{1}{2} c_{0} x^{\gamma_{e}^{+}} /\left(\gamma_{e}^{+}-1\right)\right.}{1-\exp \left\{-c_{0} x^{\gamma_{e}^{+}} /\left(\gamma_{e}^{+}-1\right)\right.}\right\} \\
& =\frac{1}{1+\exp \left\{-\frac{1}{2} c_{0} x^{\gamma_{e}^{+} /\left(\gamma_{e}^{+}-1\right)}\right\}} \\
& \geqslant \frac{1}{2} .
\end{aligned}
$$

Plugging this into (10) gives

$$
\mathrm{P}\left(W_{0}<x\right) \geqslant \frac{1}{2} \exp \left\{-c_{2} x^{\gamma_{e}^{+}} /\left(\gamma_{e}^{+}-1\right)\right\}
$$

where $c_{2}=\sup _{0<\varepsilon<1-\gamma^{+}} c_{1} c_{0}^{\gamma_{\varepsilon}^{+}}<\infty$.

Again we note that if $\gamma^{+}=\sup \log a_{0, n} / \log \mu_{0, n}$, then this result holds with $\varepsilon=0$ and $x_{0}=2 c_{1}$. 


\section{SCHRÖDER CASE}

We shall assume throughout this section that $X_{n}$ is Schröder for all $n$. The varying environment analogues to $\alpha$ are (taking any $m \geqslant 0$ )

$$
\alpha^{-}:=\liminf _{n \rightarrow \infty} \frac{\log \left(1 / p_{1}^{m, n}\right)}{\log \mu_{m, n}} \text { and } \alpha^{+}:=\limsup _{n \rightarrow \infty} \frac{\log \left(1 / p_{1}^{m, n}\right)}{\log \mu_{m, n}} \text {. }
$$

It is easily checked that $\alpha^{-}$and $\alpha^{+}$are independent of $m$, and that

$$
\liminf _{n \rightarrow \infty} \frac{\log \left(1 / p_{1}^{n}\right)}{\log \mu_{n}} \leqslant \alpha^{-} \leqslant \alpha^{+} \leqslant \limsup _{n \rightarrow \infty} \frac{\log \left(1 / p_{1}^{n}\right)}{\log \mu_{n}}
$$

Also, note that it is possible to have $\alpha^{-}<\alpha^{+}$. We shall write

$$
\alpha_{\varepsilon}^{+} \text {for } \alpha^{+}+\varepsilon \text { and } \alpha_{\varepsilon}^{-} \text {for } \alpha^{-}-\varepsilon
$$

\subsection{UPPER BOUND.}

An upper bound on the law of $W_{0}$ is obtained from an upper bound on $\varphi_{0}$, just as in the Böttcher case. As before, inequality (5) plays a central role, though some work is required to bring $p_{1}^{0, n}$ into the picture. This is done using a first term Taylor series approximation of $f_{0, n}$.

We shall need the following additions to our basic assumptions:

C4 $\bar{p}:=\sup p_{1}^{n}<1$.

C5A $\underline{p}:=\inf _{n}^{n} p_{1}^{n}>0$.

Amongst other things, $\mathrm{C} 4$ is used to guarantee $\alpha^{-}>0$.

LEMma 10. (First term approximation of $f_{m, n}$. ) Suppose that $C 1, C 4$ and C5A hold. Then for all $t_{0}<1$ and $\varepsilon>0$ there exists an $N$ such that for all $t \in\left[0, t_{0}\right]$ and $n \geqslant N+m$

$$
\begin{aligned}
f_{m, n}(t) & \leqslant t p_{1}^{m, n}(1+\varepsilon)^{(n-m) \log (1 / \bar{p})} \\
& \leqslant t p_{1}^{m, n}(1+\varepsilon)^{\log \left(1 / p_{1}^{m, n}\right)}
\end{aligned}
$$

Proof: We note to begin with that

$$
f_{m, n}(t) \stackrel{n \rightarrow \infty}{\longrightarrow} 0 \text { uniformly in } m \text { and } t \in\left[0, t_{0}\right]
$$

since (from C4)

$$
f_{n}(t) \leqslant \bar{p} t+(1-\bar{p}) t^{2}:=g(t)
$$


and thus

$$
f_{m, n}(t) \leqslant g^{(n-m)}(t) \stackrel{n \rightarrow \infty}{\longrightarrow} 0 \text { uniformly on }\left[0, t_{0}\right]
$$

where $g^{(k)}$ is the $k$-fold composition of $g$. Also

$$
f_{n}^{\prime}(t) \stackrel{t \downarrow 0}{\longrightarrow} p_{1}^{n} \quad \text { uniformly in } n
$$

since (from C1)

$$
\begin{aligned}
\left|f_{n}^{\prime}(t)-p_{1}^{n}\right| & =2 p_{2}^{n} t+3 p_{3}^{n} t^{2}+\cdots \\
& \leqslant \mu_{n} t \\
& \leqslant \bar{\mu} t .
\end{aligned}
$$

Now, as $f_{m, n}(0)=0,(11)$ will follow if we can establish that

$$
f_{m, n}^{\prime}(t) \leqslant p_{1}^{m, n}(1+\varepsilon)^{(n-m) \log (1 / \bar{p})} .
$$

Taking logarithms, this is equivalent to requiring

$$
\frac{1}{n-m} \log \frac{f_{m, n}^{\prime}(t)}{p_{1}^{m, n}} \leqslant \log (1+\varepsilon) \log (1 / \bar{p}):=\varepsilon^{\prime}
$$

which in turn is equivalent to

$$
\frac{1}{n-m} \sum_{k=m}^{n-1} \log \frac{f_{k}^{\prime}\left(f_{k+1, n}(t)\right)}{p_{1}^{k}} \leqslant \varepsilon^{\prime}
$$

For $\delta>0$ let $t_{1}$ be such that for $0 \leqslant t \leqslant t_{1}, f_{n}^{\prime}(t) \leqslant p_{1}^{n}+\delta$, and let $K$ be such that for all $t \in\left[0, t_{0}\right]$ and $n-m \geqslant K, f_{m, n}(t) \leqslant t_{1}$. That $t_{1}$ and $K$ exist follows from (13) and (12) respectively. We have

$$
\begin{aligned}
& \frac{1}{n-m} \sum_{k=m}^{n-1} \log \frac{f_{k}^{\prime}\left(f_{k+1, n}(t)\right)}{p_{1}^{k}} \\
& \quad \leqslant \frac{1}{n-m} \sum_{k=m}^{n-1-K} \log \frac{p_{1}^{k}+\delta}{p_{1}^{k}}+\frac{1}{n-m} \sum_{k=n-K}^{n-1} \log \frac{\bar{\mu}}{\underline{p}} \\
& \leqslant \frac{\delta}{\underline{p}}+\frac{K}{n-m} \log \frac{\bar{\mu}}{\underline{p}} \quad \text { (from C5A). }
\end{aligned}
$$

Choose $\delta$ so that $\delta / \underline{p} \leqslant \varepsilon^{\prime} / 2$ and $N$ such that $(K / N) \log \bar{\mu} / \underline{p} \leqslant \varepsilon^{\prime} / 2$, then the result follows. 
Proposition 11. (Algebraic upper bound for $\varphi_{0}$. ) Suppose that C1-C4 and C5A hold, then for all $0<\varepsilon<\alpha^{-}$we can find a $u_{1}$ such that for all $u>u_{1}$

$$
\varphi_{0}(u) \leqslant u^{-\alpha_{e}^{-}} \text {. }
$$

ProOF: For some (arbitrary) $u_{0}>0$ we define intervals

$$
I_{n}=\left[u_{0} \mu_{0, n}, u_{0} \mu_{0, n+1}\right) \text { for all } n \geqslant 0 \text {. }
$$

Using this $u_{0}$ in Lemma 4 gives for $u \in I_{n}, \varphi_{n}\left(u / \mu_{0, n}\right) \leqslant \beta<1$. Let $t_{0}=\beta$, then from Lemma 10 we have an $N_{1}=N_{1}(\varepsilon)$ such that for $n \geqslant N_{1}$ (that is, for $u>u_{0} \mu_{0, N_{1}}$ )

$$
\begin{array}{rlr}
\varphi_{0}(u) & =f_{0, n}\left(\varphi_{n}\left(u / \mu_{0, n}\right)\right) & \\
& \leqslant f_{0, n}(\beta) & \text { from }(6) \\
& \leqslant \beta p_{1}^{0, n}(1+\varepsilon)^{\log \left(1 / p_{1}^{0, n}\right)} \quad \text { from }(11) \\
& =\beta \mu_{0, n}^{-(1-\log (1+\varepsilon)) \log \left(1 / p_{1}^{0, n}\right) / \log \mu_{0, n}} \\
& \leqslant \beta\left(u / u_{0} \bar{\mu}\right)^{-(1-\log (1+\varepsilon)) \log \left(1 / p_{1}^{0, n}\right) / \log \mu_{0, n} .}
\end{array}
$$

Let $N_{2}=N_{2}(\varepsilon)$ be such that for $n \geqslant N_{2}$

$$
\frac{\log \left(1 / p_{1}^{0, n}\right)}{\log \mu_{0, n}} \geqslant \alpha_{\varepsilon}^{-}
$$

then for all $u>u_{0} \max \left\{\mu_{0, N_{1}}, \mu_{0, N_{2}}\right\}:=u_{1}$ we have

$$
\varphi_{0}(u) \leqslant \beta\left(u / u_{0} \bar{\mu}\right)^{-(1-\log (1+\varepsilon)) \alpha_{\bar{\epsilon}}^{-}} \leqslant \beta\left(u / u_{0} \bar{\mu}\right)^{-\alpha_{e_{*}}^{-}}
$$

where $\varepsilon_{*} \downarrow 0$ as $\varepsilon \downarrow 0$. Finally, choose $u_{0}$ to give $\beta\left(u_{0} \bar{\mu}\right)^{\alpha_{\varepsilon_{*}}^{-}}=1$ and the result follows.

PROPOSITION 12. (Algebraic upper bound on the law of $W_{0}$.) Suppose that C1-C4 and C5A hold, then there exists a $c_{1}>0$ such that for all $0<\varepsilon<\alpha^{-}$we can find an $x_{0}$ such that for all $0 \leqslant x<x_{0}$

$$
\mathrm{P}\left(W_{0}<x\right) \leqslant c_{1} x^{\alpha_{\varepsilon}^{-}} \text {. }
$$

Proof: As in the proof of Proposition 6, we get from (14) that

$$
\mathrm{P}\left(W_{0}<x\right) \leqslant e^{u x} \varphi_{0}(u) \leqslant e^{u x} u^{-\alpha_{e}^{-}} .
$$

Minimising with respect to $u$ gives at $u=\alpha_{e}^{-} x^{-1}$

$$
\begin{aligned}
\mathrm{P}\left(W_{0}<x\right) & \leqslant\left(e / \alpha_{\varepsilon}^{-}\right)^{\alpha_{\varepsilon}^{-}} x^{\alpha_{\varepsilon}^{-}} \\
& \leqslant c_{1} x^{\alpha_{e}^{-}}
\end{aligned}
$$

where $c_{1}=\sup _{0<\epsilon<\alpha^{-}}\left(e / \alpha_{e}^{-}\right)^{\alpha_{e}^{-}}<\infty$. Putting $x_{0}=\alpha_{e}^{-} u_{1}^{-1}$ gives the result. 


\subsection{LOWER BOUND.}

The lower bound is found quite easily in the Schröder case.

Proposition 13. (Algebraic lower bound for $\varphi_{0}$.) Suppose that C1-C3 hold, then there exists a $c_{1}>0$ such that for all $0<\varepsilon<\varepsilon_{0}$ ( $\varepsilon_{0}<\infty$ given) we can find a $u_{1}$ such that for all $u>u_{1}$

$$
\varphi_{0}(u) \geqslant c_{1} u^{-\alpha_{\varepsilon}^{+}}
$$

Proof: From (5) and (8) we have

$$
\begin{aligned}
\varphi_{0}(u) & \geqslant p_{1}^{0, n} \varphi_{n}\left(u / \mu_{0, n}\right) \\
& \geqslant p_{1}^{0, n} e^{-u / \mu_{0, n}} .
\end{aligned}
$$

Let

$$
\begin{aligned}
& I_{0}=[1 / \bar{\mu}, 1] \text { and } \\
& I_{n}=\left(\mu_{0, n-1}, \mu_{0, n}\right] \text { for } n>0
\end{aligned}
$$

then for $u \in I_{n}$ we have

$$
\begin{aligned}
\varphi_{0}(u) & \geqslant p_{1}^{0, n} e^{-1} \\
& =\mu_{0, n}^{-\log \left(1 / p_{1}^{0, n}\right) / \log \mu_{0, n}} e^{-1} \\
& \geqslant(u \vec{\mu})^{-\log \left(1 / p_{1}^{0, n}\right) / \log \mu_{0, n} e^{-1}}
\end{aligned}
$$

Given $\varepsilon>0$, let $N$ be such that for all $n \geqslant N$

$$
\frac{\log \left(1 / p_{1}^{0, n}\right)}{\log \mu_{0, n}} \leqslant \alpha_{\varepsilon}^{+}
$$

then for $u>\mu_{0, N-1}:=u_{1}$, we have $n \geqslant N$ and so

$$
\varphi_{0}(u) \geqslant c_{1} u^{-\alpha_{e}^{+}}
$$

where $c_{1}=\bar{\mu}^{-\alpha_{e_{0}}^{+}} e^{-1}>0$.

Note that if $\alpha^{+}=\sup _{n} \log \left(1 / p_{1}^{0, n}\right) / \log \mu_{0, n}$, then the result holds with $\varepsilon=0$ and $u_{1}=1 / \bar{\mu}$. 
Proposition 14. (Algebraic lower bound on the law of $W_{0}$.) Suppose that C1-C3 hold, then for all $0<\varepsilon<\varepsilon_{0}$ ( $\varepsilon_{0}$ given) there exists a $c_{2}>0$ and an $x_{0}$ such that for all $0 \leqslant x<x_{0}$

$$
\mathrm{P}\left(W_{0}<x\right) \geqslant c_{2} x^{\alpha_{e}^{+}} \text {. }
$$

Proof: Just as in the proof of Proposition 9, we can decompose $\varphi_{0}$ to give

$$
\begin{aligned}
\mathrm{P}\left(W_{0}<x\right) & \geqslant \frac{\varphi_{0}(u)-e^{-u x}}{1-e^{-u x}} \\
& \geqslant c_{1} u^{-\alpha_{\varepsilon}^{+}}-e^{-u x} \quad \text { from (15). }
\end{aligned}
$$

Put

$$
u=\alpha_{2 e^{x^{-1}}}^{+} \log x^{-1}
$$

then $u>u_{1}$ provided $x / \log (1 / x)<\alpha^{+} / u_{1}$. For such $x$ we get

$$
\begin{aligned}
\mathrm{P}\left(W_{0}<x\right) & \geqslant c_{1} x^{\alpha_{\varepsilon}^{+}}\left(\alpha_{2 \varepsilon}^{+} \log x^{-1}\right)^{-\alpha_{\varepsilon}^{+}}-x^{\alpha_{2 \varepsilon}^{+}} \\
& =x^{\alpha_{2 \varepsilon}^{+}}\left[c_{1} x^{-\varepsilon}\left(\alpha_{2 \varepsilon}^{+} \log x^{-1}\right)^{-\alpha_{\varepsilon}^{+}}-1\right] .
\end{aligned}
$$

Now $\left[c_{1} x^{-\varepsilon}\left(\alpha_{2 \varepsilon}^{+} \log x^{-1}\right)^{-\alpha_{\varepsilon}^{+}}-1\right] \uparrow \infty$ as $x \downarrow 0$. Thus for any $\varepsilon>0$ we can choose an $x_{0}$ such that $x<x_{0}$ implies $x / \log (1 / x)<\alpha^{+} / u_{1}$ and

$$
0<c_{2}:=\inf _{x \in\left[0, x_{0}\right]}\left[c_{1} x^{-e}\left(\alpha_{2 \varepsilon}^{+} \log x^{-1}\right)^{-\alpha_{e}^{+}}-1\right]
$$

whence the result.

\section{Mixed CASE}

Some results are still possible when $Z_{0, n}$ is neither Schröder nor Böttcher. For the purposes of this section we shall assume that $X_{n_{k}}$ is Böttcher for all $k$, where $n_{k} \uparrow \infty$ as $k \uparrow \infty$, and that all the other $X_{n}$ are either Böttcher or Schröder.

Before proceeding we shall need some more notation. Let $\tilde{f}_{k}=f_{n_{k}, n_{k+1}}$ and let $\tilde{X}_{k}$ be a random variable with this probability generating function, that is $\tilde{X}_{k} \stackrel{D}{=} Z_{n_{k}, n_{k+1}}$. Write $\tilde{\mu}_{k}$ for $\mathrm{E} \tilde{X}_{k}$, then we have the following weakenings of our basic assumptions:

$\tilde{\mathbf{C}} 1 \quad \overline{\tilde{\mu}}:=\sup _{k} \tilde{\mu}_{k}<\infty$.

C2 $\widetilde{Z}_{0, k}:=Z_{n_{0}, n_{k}}$ is uniformly supercritical.

C3 All the $\tilde{X}_{k} / \tilde{\mu}_{k}$ are dominated by some $\tilde{X}$ with $\mathrm{E} \tilde{X} \log ^{+} \tilde{X}<\infty$. 
Clearly $\tilde{X}_{k}$ is Böttcher for all $k \geqslant 0$, so we can apply our previous results for the Böttcher case directly to the current situation. Let

$$
\widetilde{\gamma}^{-}=\liminf _{k \rightarrow \infty} \frac{\log a_{n_{0}, n_{k}}}{\log \mu_{n_{0}, n_{k}}} \quad \text { and } \quad \tilde{\gamma}^{+}=\limsup _{k \rightarrow \infty} \frac{\log a_{n_{0}, n_{k}}}{\log \mu_{n_{0}, n_{k}}}
$$

and put

$$
\widetilde{\gamma}_{\varepsilon}^{+}=\widetilde{\gamma}^{+}+\varepsilon \text { and } \tilde{\gamma}_{\varepsilon}^{-}=\tilde{\gamma}^{-}-\varepsilon
$$

As before, $\tilde{\mathrm{C}} 1$ is sufficient to guarantee $\gamma^{-}>0$.

Proposition 15. (Upper bound in the mixed case.) Suppose that $\tilde{C} 1-\tilde{C} 3$ hold, then there exists a $c_{1}>0$ such that for all $0<\varepsilon<\tilde{\gamma}^{-}$we can find an $x_{0}$ such that for all $0 \leqslant x<x_{0}$

$$
\mathrm{P}\left(W_{n_{0}}<x\right) \leqslant \exp \left\{-c_{1} x^{-\tilde{\gamma}_{e}^{-} /\left(1-\tilde{\gamma}_{e}^{-}\right)}\right\}
$$

Moreover if $\tilde{\gamma}^{-}=\inf _{k} \log a_{n_{0}, n_{k}} / \log \mu_{n_{0}, n_{k}}$ then the result holds with $\varepsilon=0$.

For the lower bound we shall need the following additional assumptions:

$\tilde{\mathbf{C}} 4 \quad \overline{\tilde{p}}:=\sup _{k} p_{a_{n_{k}}, n_{k+1}}^{n_{k}, n_{k+1}}<1$, which guarantees $\widetilde{\gamma}^{+}<1$, and

Ċ5 $\tilde{Z}_{0, k}$ has uniform minimum family sizes.

Proposition 16. (Lower bound in the mixed case.) Suppose that $\tilde{C} 1-\tilde{C} 5$ hold, then there exists a $c_{2}>0$ such that for all $0<\varepsilon<1-\tilde{\gamma}^{+}$we can find an $x_{0}$ such that for all $0 \leqslant x<x_{0}$

$$
\mathrm{P}\left(W_{n_{0}}<x\right) \geqslant \frac{1}{2} \exp \left\{-c_{2} x^{-\tilde{\gamma}_{e}^{+} /\left(1-\tilde{\gamma}_{e}^{+}\right)}\right\}
$$

Moreover if $\tilde{\gamma}^{+}=\sup _{k} \log a_{n_{0}, n_{k}} / \log \mu_{n_{0}, n_{k}}$ then the result holds with $\varepsilon=0$.

Finally, note that it follows immediately from Propositions 15 and 16 that if $\tilde{\mathrm{C}} 1-\overline{\mathrm{C}} 3$ hold then

$$
\liminf _{x \rightarrow 0} \frac{\log \log \mathrm{P}\left(W_{n_{0}}<x\right)}{-\log x} \geqslant \frac{\tilde{\gamma}^{-}}{1-\tilde{\gamma}^{-}}
$$

and if $\overline{\mathrm{C}} 4$ and $\overline{\mathrm{C}} 5$ also hold then

$$
\limsup _{x \rightarrow 0} \frac{\log \log P\left(W_{n_{0}}<x\right)}{-\log x} \leqslant \frac{\tilde{\gamma}^{+}}{1-\tilde{\gamma}^{+}}
$$

Clearly, analogous inequalities can be derived from Propositions 6, 9, 12 and 14. 


\section{REFERENCES}

[1] M.T. Barlow, 'Random walks and diffusions on fractals', in Proc. Int. Congress Math. Kyoto 1990 (Springer, Tokyo, 1991), pp. 1025-1035.

[2] M.T. Barlow, 'Harmonic analysis on fractal spaces', Séminaire Bourbaki Vol. 1991/92. Astérique No 206, Exp. No 7555 (1992), 345-368.

[3] M.T. Barlow and R.F. Bass, 'The construction of Brownian motion on the Sierpinski carpet', Ann. Inst. H. Poincaré 25 (1989), 225-257.

[4] J.D. Biggins and N.H. Bingham, 'Near-constancy phenomena in branching processes', Math. Proc. Cambridge Philos. Soc. 110 (1991), 545-558.

[5] J.D. Biggins and N.H. Bingham, 'Large deviations in the supercritical branching process', Adv. Appl. Prob. 25 (1993), 757-772.

[6] P. Billingsley, Probability and measure (John Wiley and Sons, New York, 1979).

[7] N.H. Bingham, 'On the limit of a supercritical branching process', J. Appl. Probab. 25A (1988), 215-228.

[8] J.C. D'Souza and J.D. Biggins, 'The supercritical Galton-Watson process in varying environments', Stochastic Process. Appl. 42 (1992), 39-47.

[9] S. Dubuc, 'La densité de la loi-limite d'un processus en cascade expansif', Z. Wahrschein 19 (1971), 281-290.

[10] S. Dubuc, 'Problèmes relatifs a l'itération des fonctions suggerés par les processus en cascade', Ann. Inst. Fourier 21 (1971), 171-251.

[11] D. Fearn, 'Galton-Watson processes with generation dependence', in Proc. 6th Berkeley Symposium on Math. Stat. and Prob. 4, 1971, pp. 159-172.

[12] R.T. Goettge, 'Limit theorems for the supercritical Galton-Watson process in varying environments', Math. Biosci. 28 (1975), 171-190.

[13] B. Hambly, 'On the limiting distribution of a supercritical branching process in a random environment', J. Appl. Probab. 29 (1992), 499-518.

[14] B. Hambly, 'On constant tail behaviour for the limiting random variable in a supercritical branching process', (preprint, 1994).

[15] P. Jagers, 'Galton-Watson process in varying environments', J. Appl. Probab. 11 (1974), 174-178.

[16] H. Kesten and B. Stigum, 'A limit theorem for multidimensional Galton-Watson processes', Ann. Math. Stat. 37 (1966), 1211-1223.

[17] I.M. MacPhee and H.J. Schuh, 'A Galton-Watson branching process in varying environments with essentially constant means and two rates of growth', Austral. J. Stat. 25 (1983), 329-338.

School of Mathematics and Statistics

University of Sheffield

Sheffield S10 2UN

United Kingdom 